\title{
Solvation Stokes-Shift Dynamics Studied by Chirped Femtosecond Laser Pulses
}

\author{
Arkaprabha Konar, ${ }^{\dagger}$ Vadim V. Lozovoy, ${ }^{\dagger}$ and Marcos Dantus, ${ }^{* \dagger, \dagger}$ \\ †Department of Chemistry, Michigan State University, East Lansing, Michigan 48824, United States \\ ${ }^{*}$ Department of Physics and Astronomy, Michigan State University, East Lansing, Michigan 48824, United States
}

Supporting Information

ABSTRACT: The early optical dynamic response, resulting population, and electronic coherence are investigated experimentally and modeled theoretically for IR144 in solution. The fluorescence and stimulated emission response are studied systematically as a function of chirp. The magnitude of the chirp effect on fluorescence and stimulated emission is found to depend quadratically on pulse energy, even where excitation probabilities range from 0.02 to $5 \%$, in the so-called "linear excitation regime". Interestingly, the shape of the chirp dependence on fluorescence and stimulated emission is found to be independent of pulse energy. The chirp dependence reveals dynamics related to solvent rearrangement following excitation and also depends on electronic relaxation of the chromophore. The experimental results are successfully simulated using a four-level model in the presence of inhomogeneous broadening of the electronic transitions.

SECTION: Spectroscopy, Photochemistry, and Excited States

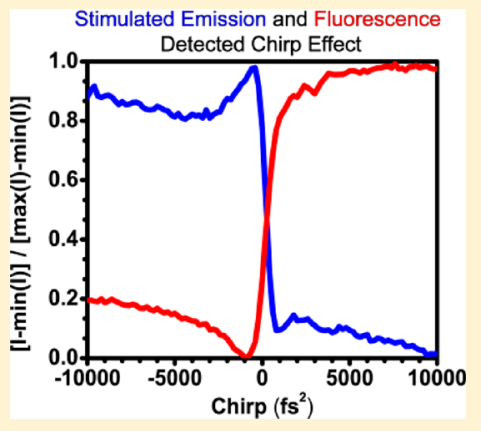

$\mathrm{R}$ apid developments in the field of femtosecond lasers along with the development of multidimensional spectroscopic techniques aided by theoretical modeling have inspired numerous studies pertaining to the elucidation of vibrational and electronic coherences in a wide range of quantum systems. New insights into the excited state dynamics and energy transfer mechanisms of dye molecules, ${ }^{1}$ macromolecules, ${ }^{2}$ and light harvesting photosynthetic aggregates ${ }^{3}$ have been obtained. Evidence of long-lived coherences in biological systems even at physiological temperatures ${ }^{4,5}$ has provided an impetus to the already hot field of quantum coherence and its role in biological systems. Solvation of excited states, ${ }^{6,7}$ relaxations, and decoherence have emerged as key aspects to that need to be measured and understood.

Spectral phase modulation has proven to be a powerful tool in the coherent control community for controlling condensed phase systems. Phase shapes ranging from sinusoidal ${ }^{8}$ to chirp modulations ${ }^{9,10}$ have been extensively investigated. Order of magnitude enhancement in the concerted elimination pathway leading to $\mathrm{I}_{2}$ product formation in the photodissociation reaction of $\mathrm{CH}_{2} \mathrm{I}_{2}$ by the use of positively chirped $312 \mathrm{~nm}$ femtosecond laser pulses has been demonstrated by Pastirk et al. ${ }^{11}$ Quadratic phase on a pulse (chirp) has been also successful in selectively exciting coherent wave packet motion in the ground and excited state along with achieving complete population transfer between electronic states. Shank and coworkers observed the effect of chirped pulses on the fluorescence intensity of LC690 and explained this effect using the wave packet following hypothesis, ${ }^{12}$ which was followed by several experimental and theoretical papers investigating the chirp effect on resonant electronic transition in different systems while continuing to develop the wave packet following model. ${ }^{13-16}$ Cao et al. also developed a simple intrapulse three-level model to explain the chirp effect on fluorescence. $^{14}$ The role of stimulated transitions without relaxation was discussed by Hashimoto et al., ${ }^{17}$ while the competition between stimulated transition and relaxation have been discussed by Bardeen et al. ${ }^{18}$ Fainberg and co-workers, on the other hand, have tried to include non-Markovian relaxation in the existing theory for ultrafast excitation and chirp effects. ${ }^{19-23}$ The response to time inversion of shaped femtosecond pulses was investigated for various nonlinear processes, and it was found that processes taking place on a very fast time scale $(0-20 \mathrm{fs})$ resulted in a symmetric response, while asymmetry was found in processes taking place on a longer time scale. ${ }^{24}$ Dynamic Stark shift and irreversible population loss upon chirped adiabatic passage in a two-state quantum system has been theoretically analyzed as well to explain the asymmetry of the chirp effect. ${ }^{25}$ Solvation is responsible for the fast relaxation and rapid loss of coherence due to the interaction of the molecules with the surrounding solvent molecules.

Coherent control of large molecules in solution within the linear laser intensity regime of excitation is also intrinsically related to the solvation induced decoherence and has been investigated in the isomerization of the retinal chromophore in bacteriorhodopsin ${ }^{26,27}$ as well as for dye molecule, coumarin, in nonpolar solvents. ${ }^{28}$ By varying the solvent viscosity, van der Walle et al. ${ }^{28}$ linked viscosity to the rapid loss of coherence, which limits the action of the pulse on the molecule. Similar

Received: June 12, 2012

Accepted: August 10, 2012 

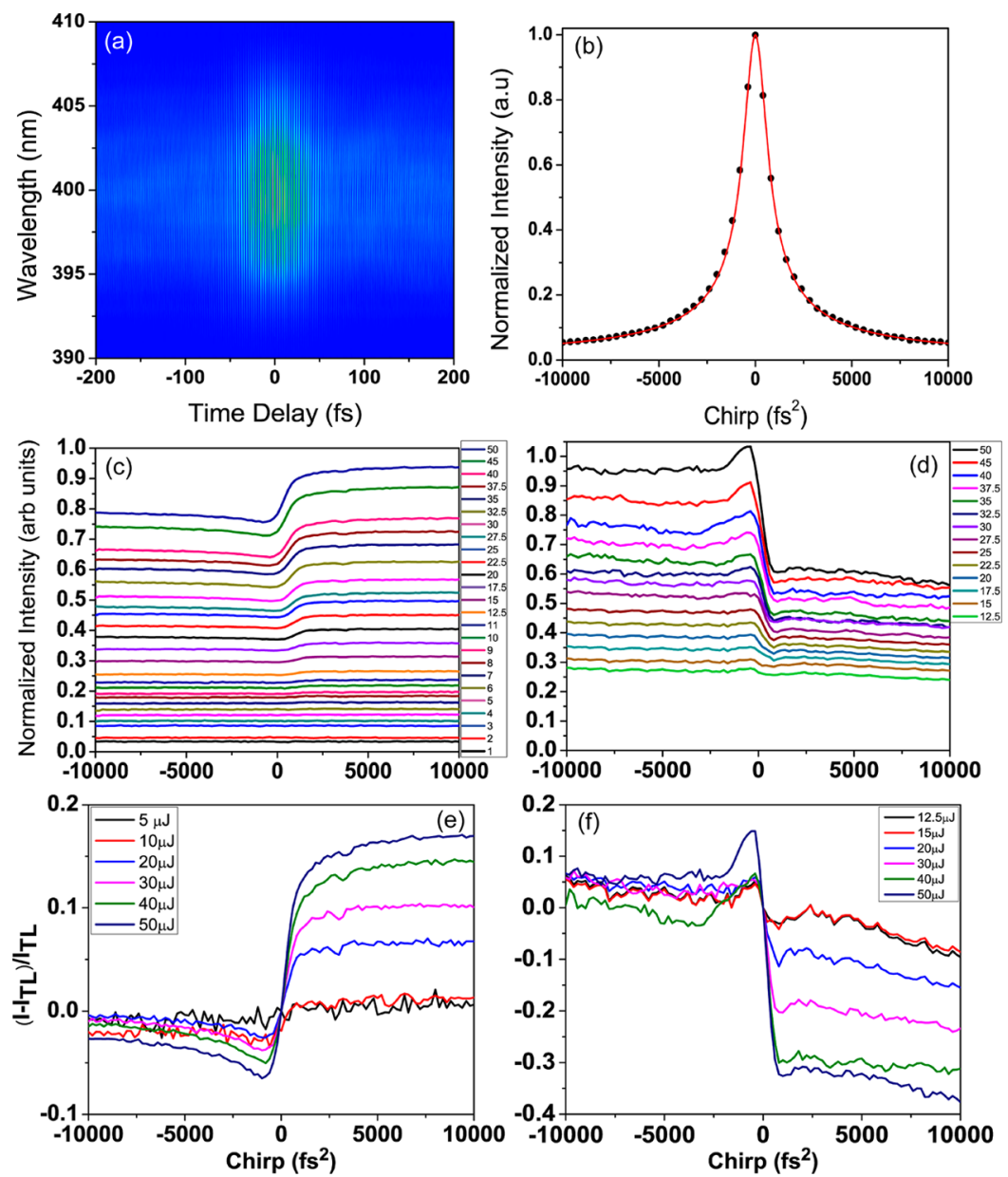

Figure 1. (a) Experimental interferometric XFROG trace for the transform limited 36 fs pulses. (b) Experimental data (black dots) for SHG Chirp scan along with the theoretically simulated curve (red line). Dependence of integrated intensity of fluorescence (c) and stimulated emission (d) as a function of chirp at different intensities of the laser pulse. Difference between fluorescence measured at different chirp values normalized to TL pulse excitation as a function of chirp for (e) fluorescence and (f) stimulated emission.

solvent and viscosity dependence was also observed on the chirp dependence of the fluorescence by our group and will be reported in a subsequent publication. Ruhman and coworkers $^{29}$ have presented a multimode perspective to the problem of chirped excitations in condensed phase, where each mode responds to excitation chirp independently, leading to mode-specific optimal chirp leading to the entanglement of the dynamics of excitation in different modes. Katz et al..$^{30}$ have theoretically demonstrated the weak field control of a branching reaction by using chirp as the control knob and discussed the role of the dissipative environment in stabilizing the outcome in a short time scale in the linear regime of excitation.

IR 144 has been the subject of various studies because of its high absorption cross section at $800 \mathrm{~nm}$ and its large solventdependent Stokes shift. Dynamic absorption methods were used to project the phase relationships between the coherent wave packet motions on the ground-state and excited-state potential-energy surfaces. ${ }^{31}$ Pump-probe investigations performed by $\mathrm{Yu}$ et $\mathrm{al}^{32}$ focused on the solvatochromatic behavior of the cyanine dyes. Coherence-period resolved transient grating was also used to measure the solvation dynamics of IR144. ${ }^{33}$ Due to the prevalence of IR-144 data in the literature, the fluorophore serves as an ideal model system for investigating the contributions of different experimental designs to early optical response. All the above-mentioned theories until now have been partially successful in explaining some of the experimental effects, mainly the fact that fluorescence with positively chirped pulses was stronger than the negatively chirped pulses, while using very different physical models. An effort has been made in this letter to investigate the role of chirped femtosecond pulses on fluorescent dye molecules in solution, which leads one to the elucidation of some simple but very important pieces of the puzzle under the broad area encompassing weak field coherent control and Stokes shift in solvated dye molecules.

The experiments carried out involved a single shaped laser pulse and the collection of fluorescence (spontaneous emission collected at right angles) and stimulated emission (coherent light detected collinear with the laser) as a function of chirp in the excitation pulse. Note that the stimulated emission results from the single pulse used for excitation; there is no probe pulse causing the emission. Theoretical analysis using a four level system with homogeneous relaxation and inhomogeneous broadening further strengthens our hypothesis that the stimulated emission results from an ensemble of molecules with unrelaxed electronic coherence between the ground and excited states similar to the free induction decay in nuclear magnetic resonance studies. The quadratic nature of the macroscopic polarization was further confirmed by the 

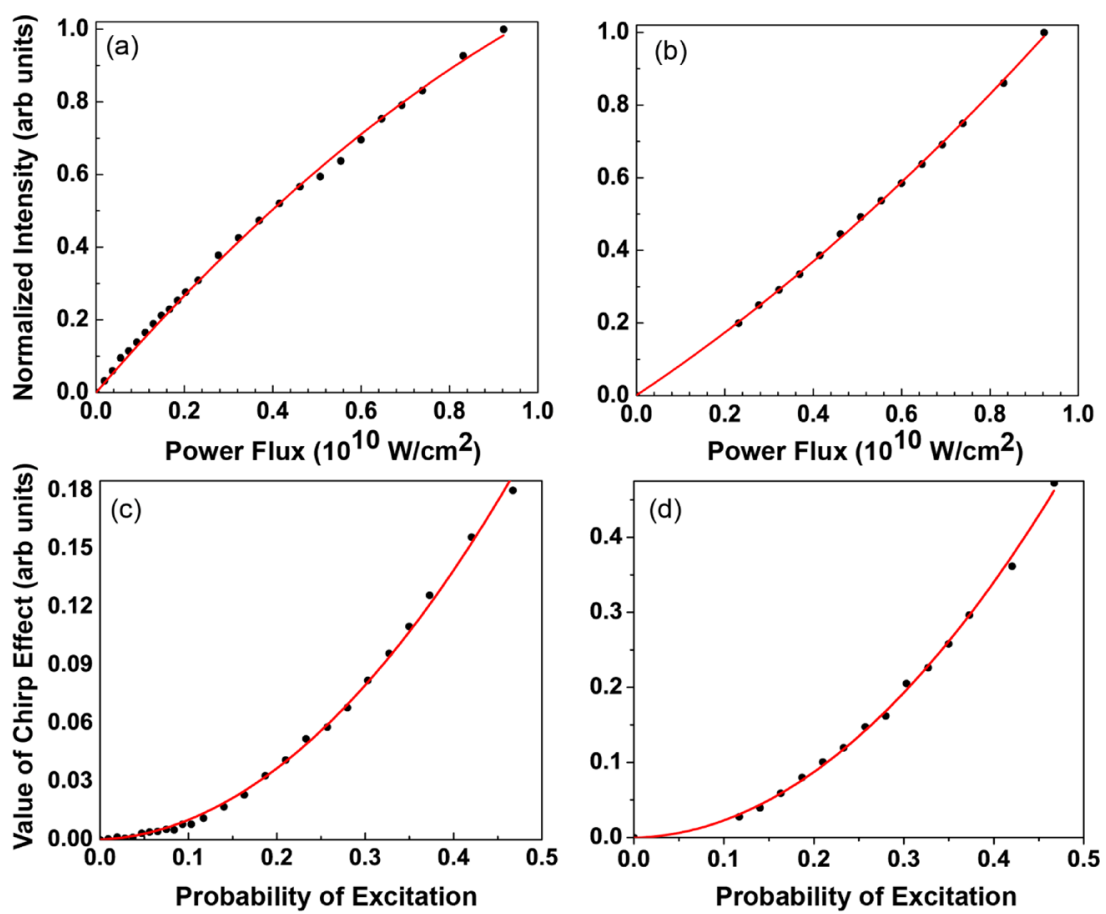

Figure 2. Dependence of integrated intensity for (a) fluorescence and (b) stimulated emission as a function of average laser power flux for the TL excitation. The value of chirp effect as a function of calculated probability of one photon excitation with TL pulse are shown for (c) fluorescence and (d) stimulated emission. The $y$-axis is the difference between the maximum and minimum of the value measured at different pulse energies. The red curves represent quadratic fits to the experimental data.

quadratic concentration dependence (Supporting Information Figure S3). In addition, this model successfully explains the shape of the chirp effect for both the population and coherence.

We performed a power dependence study while tracking the fluorescence and stimulated emission as a function of linear chirp in order to establish a definitive ruler as to what the scientific community calls the "linear regime" and to explore where deviations from the linear regime are observed. Another important motivation behind carrying out the power dependence was to assess the magnitude of the effect at different probabilities of excitation upon chirp modulation. The present study was performed in the excitation range of $0.02-45 \%$ corresponding to a maximum fluence of $10^{14}$ photons $/ \mathrm{cm}^{2}$.

The electric field in the frequency domain is represented as $E(\omega)=|E(\omega)| \exp [i \varphi(\omega)]$, where the phase term $\phi(\omega)$ can be expanded as $\phi(\omega)=\phi\left(\omega_{0}\right)+\phi^{\prime}(\omega)\left(\omega-\omega_{0}\right)+(1 /$ 2) $\phi^{\prime \prime}(\omega)\left(\omega-\omega_{0}\right)^{2}+\ldots ., \phi^{\prime \prime}$ is referred to as the chirp on the phase. The amplified pulse is compressed using the MIIPS algorithm (as discussed in the Experimental Methods section), and a corresponding i-XFROG plot of the transform limited (TL) pulse is shown in Figure 1a. To demonstrate the accuracy with which the calibrated pulse shaper is able to deliver phase modulated pulses at the sample, we performed a chirp scan while monitoring the SHG at $400 \mathrm{~nm}$. The experimental points (black dots) have been plotted together with the theoretically predicted values of the integrated SHG intensity (red line) as shown in Figure 1b. The shaper accuracy is seen from the close match between the experimental curve and the simulated values. The chirp dependence of the SHG intensity is symmetric and has no distortions that could affect the observed chirp-dependence curves.

The chirp dependence of fluorescence and stimulated emission of IR 144 are respectively shown in Figure 1. Enhancement of the fluorescence relative to the TL pulses is observed for positively chirped pulses as expected from the previous studies, while the opposite behavior is observed for the stimulated emission as the negatively chirped pulses enhance this emission. The magnitude of enhancement of the stimulated emission is $\sim 40 \%$ as compared to $\sim 15 \%$ enhancement in fluorescence for the highest excitation power. This result is also in accordance to the recently observed out-of-phase behavior of fluorescence and stimulated emission when using pairs of noninterfering pulse replicas. ${ }^{34}$ An intensity-dependent study reveals the gradual dependence of the shape of the curve from the excitation intensity, as evident from Figure $1 c, d$. We define the chirp effect as the normalized difference between the observed signal with chirped and TL pulses for chirp values from $-10000 \mathrm{fs}^{2}$ to $10000 \mathrm{fs}^{2}$. This is done in order to create a reference point to compare the curves corresponding to different excitation energies. This is plotted for the different excitation powers in Figure 1e,f.

The intensity dependence of fluorescence and stimulated emission for TL pulses are shown in Figure 2a,c. Unfocused 36 fs (fwhm) TL pulses were used to acquire the data. The pulse duration corresponding to a $10000 \mathrm{fs}^{2}$ chirped pulse is determined to be $770 \mathrm{fs}$. The sample irradiated area is $\sim 23$ $\mathrm{mm}^{2}$ and the maximum and minimum pulse energies used during the study are $0.01 \mu \mathrm{J}$ and $50 \mu \mathrm{J}$ corresponding to peak powers of $10^{6}$ and $10^{10} \mathrm{~W} / \mathrm{cm}^{2}$ for transform limited pulses and $10^{4}$ and $10^{8} \mathrm{~W} / \mathrm{cm}^{2}$ for $10000 \mathrm{fs}^{2}$ chirped pulses, respectively. Deviation from linearity is observed at higher powers with saturation of fluorescence. The overall behavior of the intensity dependence under TL excitation can be more clearly understood if we recalculate the $y$ axis from normalized intensity to the total value of the effect calculated as the difference of the maximum and the minimum value (after normalizing the values corresponding to different pulse energies) of the observed signal intensity and is plot as a 
function of probability of excitation, as shown in Figure 2b,d. The probability of excitation $P$ is calculated as $P=F \sigma$ where $F=$ $I t / h \nu$ is the number of photons per square centimeter, and $\sigma$ is the absorption cross section at $800 \mathrm{~nm}$, calculated to be $2.6 \times$ $10^{-16} \mathrm{~cm}^{2}$.

The magnitude of the chirp effect is quadratic for both the fluorescence and stimulated emission. This is a clear signature that the observed effect is nonlinear in nature and that it disappears at low intensity for weak field interactions. The quadratic dependence means that it is a two-photon, pumpdump effect. Upon normalizing the curves in Figure $1 a, b$, the shape and overall behavior of the dependence becomes very clear. The minimum value of the effect, $\min (I)$ was subtracted from each curve and is normalized on the difference between maximum, $\max (I)$, and minimum of the signals. This value $[\mathrm{I}-$ $\min (I)] /[\max (I)-\min (I)]$ has an overall size of 1 , as seen from Figure 3. The fluorescence and stimulated emission

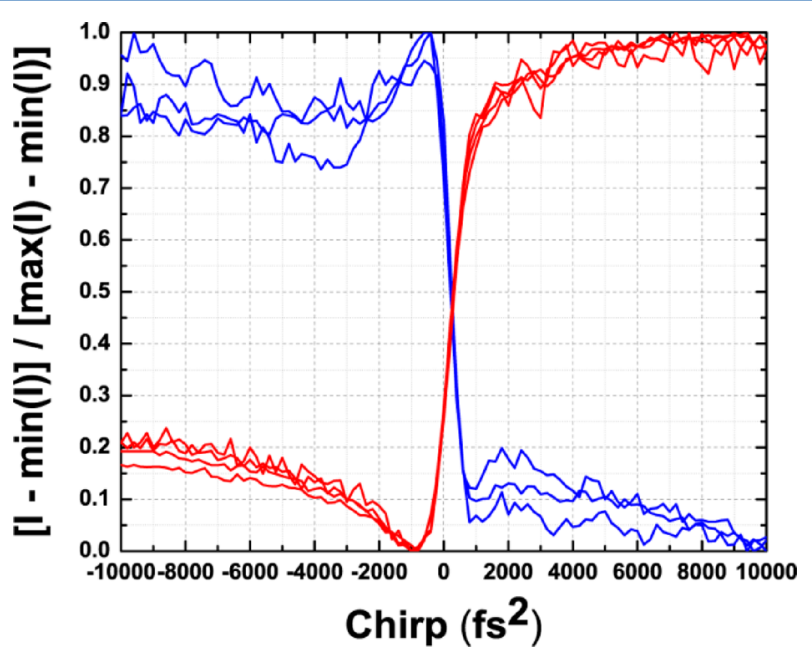

Figure 3. Normalized fluorescence detected (red) and stimulated emission detected (blue) chirp effect for different laser intensities.

detected chirp effect for $3.5 \times 10^{-4} \mathrm{~J} / \mathrm{cm}^{2}, 2.8 \times 10^{-4} \mathrm{~J} / \mathrm{cm}^{2}, 2.1$ $\times 10^{-4} \mathrm{~J} / \mathrm{cm}^{2}$ and $1.4 \times 10^{-4} \mathrm{~J} / \mathrm{cm}^{2}$ are plotted in red and blue, respectively. It is important to note that the magnitude of the total effect and shape of the chirp effect is practically independent from the energy of the pulse, which is a clear signature that the mechanism behind this effect does not depend on the laser power.

The simplest theoretical model capable of reproducing the observed data is a four-level model having two ground and two excited states. A schematic representing the model is shown in Figure 4. The irreversible relaxation from the second excited state to the first excited state is shown as a dashed arrow.

Since the experiments clearly point towards the nonlinear nature of the observed effect (see Supporting Information), we use Liouville equation to model the system

$$
\begin{aligned}
\frac{\mathrm{d} \rho_{a a}}{\mathrm{~d} t}= & -\frac{i}{\hbar} \Sigma_{\nu}\left(V_{a \nu} \rho_{\nu a}-\rho_{a \nu} V_{\nu a}\right)+\Sigma_{E b>E a} \Gamma_{a b} \rho_{b b} \\
& -\Sigma_{E b<E a} \Gamma_{b a} \rho_{a a} \\
\frac{\mathrm{d} \rho_{a b}}{\mathrm{~d} t}= & -i\left(\omega_{a}-\omega_{b}\right) \rho_{a b}-\frac{i}{\hbar} \Sigma_{\nu}\left(V_{a \nu} \rho_{\nu b}-\rho_{a \nu} V_{\nu b}\right)-\gamma_{a b} \rho_{a b}
\end{aligned}
$$

where $a$ or $b$ represent the states of the system, $\rho_{a a}$ and $\rho_{a b}$ are the diagonal and the off-diagonal elements of the density matrix

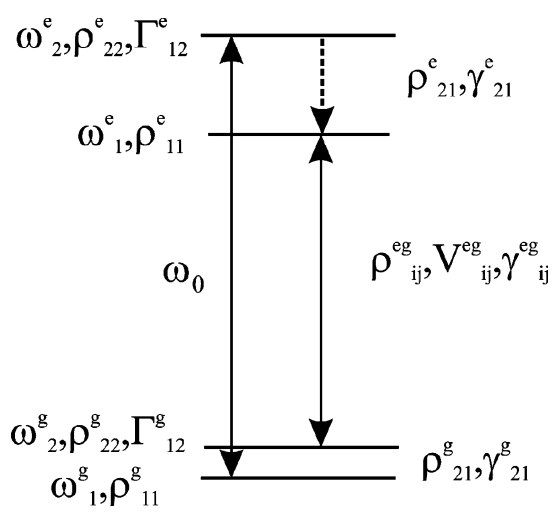

Figure 4. Schematic of the four-level model used to simulate the experimental results. $\omega_{0}$ represent the transition frequency, $\omega$ is the carrier frequency of the pulse, $V$ is the dipole interaction of transitions with light, $\Gamma$ is the relaxation rates in ground and excited states, $\gamma$ values are the dephasing rates, and $\rho$ values are elements of density matrices representing the population and the coherences.

representing populations of the states and coherences between the states respectively. The dephasing rate of the coherence is $\gamma_{a b}=1 / 2\left(\Gamma_{a}+\Gamma_{b}\right)$, where $\Gamma_{a}$ and $\Gamma_{b}$ are relaxation rates of the populations of states $a$ and $b$. The matrix element of dipole interaction with chirped pulse is represented as

$$
V_{a b}(t)=\mu_{a b} E_{0} \sqrt{\frac{\tau_{0}^{2}}{\tau_{0}^{2}-i \phi^{\prime \prime}}} \exp \left[-\frac{1}{2} \frac{t^{2}}{\tau_{0}^{2}-i \phi^{\prime \prime}}\right]
$$

where $\mu_{a b}$ is the corresponding matrix element of interaction, $\tau_{0}$ is the duration of the TL pulse, and $\phi^{\prime \prime}$ is the value of scanned chirp.

We numerically solve the system of differential equations for the density matrix without the condition of weak field interaction; pulse energies corresponding to the deviation from linearity and saturation of population transfer were used in the simulations. The diagonal elements of the density matrix $\rho_{11}^{e}$ (see Figure 4) corresponding to the lower excited state at later times (when the pulse is over and relaxations are finished) is proportional to the observed fluorescence. The best fit between theory and experiment (see Figure 5) was found when the stimulated emission was considered to be the integrated absolute value square of the off diagonal elements of the density matrix $\rho^{\text {eg }}{ }_{12}$ (see Figure 4) between the lowest exited state and highest ground state.

We assign the stimulated emission as the light emitted by the macroscopic polarization of the sample, which is a coherent sum of the electromagnetic waves emitted by the microscopic coherences of dye molecules described by the off diagonal elements of density matrix. The quadratic dependence of the intensity of stimulated emission with respect to concentration is a clear signature that the homodyne-detected signal originated from coherent polarization of the solution (see Supporting Information)

Position of the second ground state was kept close to the lowest level when the corresponding period of vibrations $T_{\mathrm{g}}=$ $2 \pi /\left(\omega_{2}^{\mathrm{g}}-\omega_{1}^{\mathrm{g}}\right)$ is longer than the pulse duration or relaxation/ dephasing times. The dephasing rate of off-diagonal matrix elements $\rho_{21}^{\mathrm{e}}$ and $\rho^{\mathrm{g}}{ }_{21}$ are the sum of two contributions: $1 / 2$ of relaxation rate and pure "collisional" dephasing rate induced by solvent $\gamma_{\mathrm{c}}$. In our case $\gamma^{\mathrm{g}}{ }_{21}=(1 / 2) \Gamma^{\mathrm{g}}{ }_{12}+\gamma^{\mathrm{g}}{ }_{\mathrm{c}}$ and $\gamma_{21}^{\mathrm{e}}=(1 /$ 2) $\Gamma_{12}^{\mathrm{e}}+\gamma_{\mathrm{c}}^{\mathrm{e}}$. The ground state relaxation time, $\tau_{\mathrm{g}}=1 / \Gamma_{12}^{\mathrm{g}}$ is long compared to the duration of the pulse or relaxation/ 

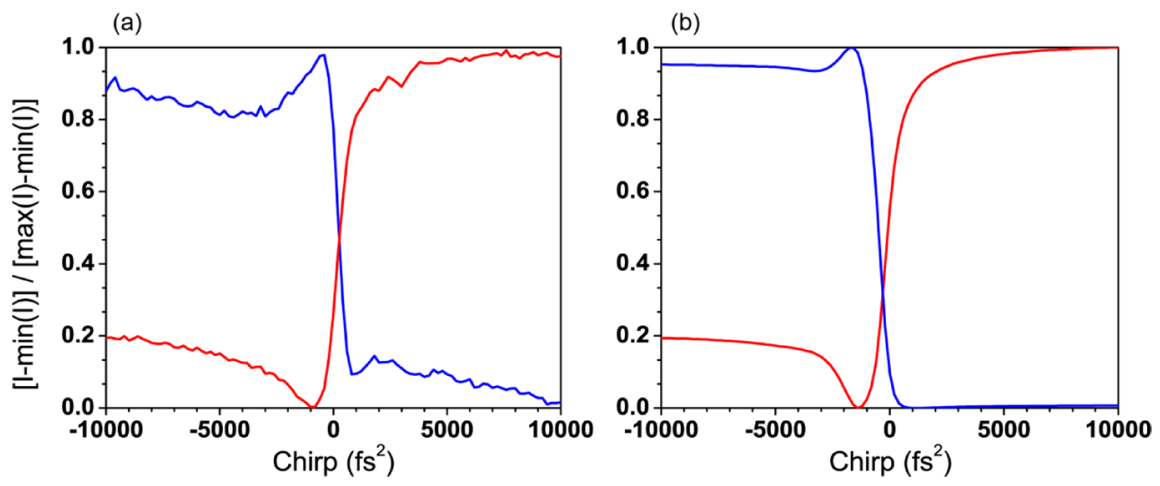

Figure 5. Normalized plots of (a) experimentally determined and (b) theoretically simulated chirp dependence of fluorescence (red) and stimulated emission (blue). Experimental curves are chirp effects averaged over inhomogeneous broadening of absorption and emission lines.

dephasing times. We have found that the best reproduction of the experimental results is obtained when the relaxation rate from the upper excited state to the lower one $\tau_{\mathrm{e}}=1 / \Gamma_{12}^{\mathrm{e}}$ is comparable or even faster than the TL pulse duration $\tau_{\mathrm{p}}$ (when the value when intensity of pulse drops in $e^{2}$ times, in our case $21 \mathrm{fs})$. We also found that to reproduce the experimental chirp effect in the model we should put the relaxation rate from the second excited state to the lower excited state $\tau_{\mathrm{c}}=\Gamma_{12}^{\mathrm{e}}$, faster than the collisional dephasing $\gamma_{c}^{\mathrm{e}}$. Therefore to summarize the above-mentioned findings we can state that the characteristic times in the model should satisfy the conditions $\tau_{\mathrm{e}} \leq \tau_{\mathrm{p}} \ll \tau_{\mathcal{c}}$ or relaxation time from the directly excited unrelaxed upper electronic state to the lower fluorescent state is comparable or shorter than the duration of the pulse, and collisional dephasing time should be longer than the pulse duration. In the result presented in Figure 5, $\tau_{\mathrm{e}}=20 \mathrm{fs}$ and $\tau_{\mathrm{g}}=100 \mathrm{fs}$ are used, although very close simulated curves are obtained for $\tau_{\mathrm{e}}=20 \mathrm{fs}$, $\tau_{\mathrm{c}}=200 \mathrm{fs}, T_{\mathrm{g}}=1000 \mathrm{fs}$, and $\tau_{\mathrm{g}}=10000 \mathrm{fs}$, reflecting the fact that the most critical condition is the fast relaxation between excited states and relatively slow relaxation or dephasing from the lower excited state and even slower dynamics in the ground state. Physically it means that solvation dynamics is faster than dephasing of the polarization.

In our model, we put $V_{12}=V_{21}$ and $V_{11}=V_{22}=0$ (see Figure 4); these conditions reflect the idea that the lowest ground state is coupled with the upper state, but the relaxed excited state is coupled with a different ground state. Our model is also able to correctly reproduce the independence of the shape of the chirp effect from the energy of the pulse (see Figure 3) and therefore does not depend on the exact value of the amplitude of the electric field.

It has also been found that the best reproduction of the experimental curves (fluorescence and stimulated emission) is obtained when inhomogeneous broadening is taken into account. This is included in the model of the upper and lower states in a very particular way. We use sets of homogeneous absorption lines to fill the actual absorption line, and for each absorption profile (taken from experiment), we have a corresponding line in the fluorescence spectral profile, and it is very important for these lines to be symmetrical with respect to the center point between the two profiles, or physically speaking, the more energetically distorted line in the inhomogeneous spectrum is more distorted in the solvated state. Experimental absorption, fluorescence, stimulated emission spectra, and an example of the filling of inhomogeneous profiles with homogeneous states are shown in the Supporting Information. We should mention that results of simulation are sensitive to the line positions in the region where absorption and emission spectra overlap with the laser spectrum. Our fourlevel model closely reproduces the experimental results and also has a physical sense of very fast motion from the upper excited state to the lower one. The relaxation from the excited state to the fluorescent state can be treated as the dynamical Stokes shift that occurs so fast that there is no time to reach equilibrium with the solvent.

We have experimentally analyzed the chirp dependence of the stimulated emission form dilute dye molecules for the first time. We measured the chirp dependence on fluorescence and stimulated emission intensities at different pulse energies and found the value of chirp effect in both emissions to be quadratic with respect to the energy of the pulses. This is a clear signature that the phase-dependent emission results from a two-photon nonlinear optical process, even for very low excitation probabilities (see Supporting Information showing this dependence from excitation probability ranging from 0.0002 to 0.06 ). This observation is significant, and should be considered when discussing phase control experiments in the "linear regime". We have also found that the shape of the chirp dependence as a function of chirp value is independent of pulse energy. Theoretically, we have formulated a model that describes the experimental results very well. In this model we use minimum number of parameters to match the experimental result. The time scale of Stokes shift from the excited state to the fluorescent state should be approximately equal to the TL pulse duration and faster than dephasing by solvent. We also found that inhomogeneous broadening plays an important role, and it helps in the improved agreement of the simulations with the experimental data.

\section{EXPERIMENTAL METHODS}

The femtosecond laser system consists of a femtosecond amplified laser (Spitfire, Spectral physics). A pulse shaper (MIIPS BOX 640, Biophotonic Solutions Inc.) adaptively corrects high-order dispersion of the laser pulses and allows us to introduce exact chirp values at the sample. The system uses the MIIPS algorithm for measuring and compressing the pulses. ${ }^{35,36}$ Experimental measurements included chirp scans performed by the pulse shaper, where the phase on the pulse introduced $\phi(\omega)=(1 / 2) \phi^{\prime \prime}\left(\omega-\omega_{0}\right)^{2}$, where $\phi^{\prime \prime}$ was varied from -10000 to $10000 \mathrm{fs}^{2}$. The fluorescence and forward emission signals were collected simultaneously using a spectrometer (USB 4000, Ocean Optics). A $10^{-6} \mathrm{M}$ solution of IR144 in methanol was used as the sample at room temperature. The dye IR144 was purchased from Exciton and 
used without further purification. The beam spot size (when intensity drops to $1 / e^{2}$ ) is $2.7 \mathrm{~mm}$ and was measured using a charge-coupled device (CCD) beam profiler (Coherent).

\section{ASSOCIATED CONTENT}

\section{S Supporting Information}

A short discussion on the experimental setup along with the experimental spectra, the magnitude of the chirp effect as a function of intensity and the concentration dependence of the forward emission are presented. Interferometric XFROG measurements for chirped pulses have also been included. This material is available free of charge via the Internet at http://pubs.acs.org

\section{AUTHOR INFORMATION}

\section{Corresponding Author}

*Address: Chemistry Building, 578 S. Shaw Lane, Michigan State University, East Lansing, MI 48824. E-mail: dantus@msu. edu.

\section{Notes}

The authors declare no competing financial interest.

\section{ACKNOWLEDGMENTS}

We thank DOE SISGR (DE-SC0002325), Dr. Jeff Krause Program Manager, for support of this research. Useful discussions with Prof Shaul Mukamel are also sincerely acknowledged. Valuable comments and suggestions from the reviewers are highly acknowledged.

\section{REFERENCES}

(1) Fidler, A. F.; Harel, E.; Engel, G. S. Dissecting Hidden Couplings Using Fifth-Order Three-Dimensional Electronic Spectroscopy. J. Phys. Chem. Lett. 2010, 1, 2876-2880.

(2) Collini, E.; Scholes, G. D. Coherent Intrachain Energy Migration in a Conjugated Polymer at Room Temperature. Science 2009, 323, 369-373.

(3) Collini, E.; Wong, C. Y.; Wilk, K. E.; Curmi, P. M. G.; Brumer, P.; Scholes, G. D. Coherently Wired Light-Harvesting in Photosynthetic Marine Algae at Ambient Temperature. Nature 2010, 463, 644-U69.

(4) Engel, G. S.; Calhoun, T. R.; Read, E. L.; Ahn, T. K.; Mancal, T.; Cheng, Y. C.; Blankenship, R. E.; Fleming, G. R. Evidence for Wavelike Energy Transfer through Quantum Coherence in Photosynthetic Systems. Nature 2007, 446, 782-786.

(5) Panitchayangkoon, G.; Hayes, D.; Fransted, K. A.; Caram, J. R.; Harel, E.; Wen, J. Z.; Blankenship, R. E.; Engel, G. S. Long-Lived Quantum Coherence in Photosynthetic Complexes at Physiological Temperature. Proc. Natl. Acad. Sci. U.S.A. 2010, 107, 12766-12770.

(6) Fleming, G. R.; Cho, M. H. Chromophore-Solvent Dynamics. Annu. Rev. Phys. Chem. 1996, 47, 109-134.

(7) Stratt, R. M.; Maroncelli, M. Nonreactive Dynamics in Solution: The Emerging Molecular View of Solvation Dynamics and Vibrational Relaxation. J. Phys. Chem. 1996, 100, 12981-12996.

(8) Lozovoy, V. V.; Pastirk, I.; Walowicz, K. A.; Dantus, M. Multiphoton Intrapulse Interference. II. Control of Two- and ThreePhoton Laser Induced Fluorescence with Shaped Pulses. J. Chem. Phys. 2003, 118, 3187-3196.

(9) Nibbering, E. T. J.; Wiersma, D. A.; Duppen, K. Ultrafast Nonlinear Spectroscopy with Chirped Optical Pulses. Phys. Rev. Lett. 1992, 68, 514-517.

(10) Bardeen, C. J.; Yakovlev, V. V.; Wilson, K. R.; Carpenter, S. D.; Weber, P. M.; Warren, W. S. Feedback Quantum Control of Molecular Electronic Population Transfer. Chem. Phys. Lett. 1997, 280, 151-158.

(11) Pastirk, I.; Brown, E. J.; Zhang, Q. G.; Dantus, M. Quantum Control of the Yield of a Chemical Reaction. J. Chem. Phys. 1998, 108, 4375-4378.
(12) Cerullo, G.; Bardeen, C. J.; Wang, Q.; Shank, C. V. High-Power Femtosecond Chirped Pulse Excitation of Molecules in Solution. Chem. Phys. Lett. 1996, 262, 362-368.

(13) Bardeen, C. J.; Yakovlev, V. V.; Squier, J. A.; Wilson, K. R. Quantum Control of Population Transfer in Green Fluorescent Protein by Using Chirped Femtosecond Pulses. J. Am. Chem. Soc. 1998, 120, 13023-13027.

(14) Cao, J. S.; Che, J. W.; Wilson, K. R. Intrapulse Dynamical Effects in Multiphoton Processes: Theoretical Analysis. J. Phys. Chem. A. 1998, 102, 4284-4290.

(15) Misawa, K.; Kobayashi, T. Wave-Packet Dynamics in a Cyanine Dye Molecule Excited with Femtosecond Chirped Pulses. J. Chem. Phys. 2000, 113, 7546-7553.

(16) Vogt, G.; Nuernberger, P.; Selle, R.; Dimler, F.; Brixner, T.; Gerber, G. Analysis of Femtosecond Quantum Control Mechanisms with Colored Double Pulses. Phys. Rev. A. 2006, 74, 3.

(17) Hashimoto, N. T.; Misawa, K.; Lang, R. Three-Level Picture for Chirp-Dependent Fluorescence Yields under Femtosecond Optical Pulse Irradiation. Appl. Phys. Lett. 2003, 82, 2749-2751.

(18) Bardeen, C. J.; Cao, J. S.; Brown, F. L. H.; Wilson, K. R. Using Time-Dependent Rate Equations to Describe Chirped Pulse Excitation in Condensed Phases. Chem. Phys. Lett. 1999, 302, 405-410.

(19) Fainberg, B. D. Nonlinear Polarization and Spectroscopy of Vibronic Transitions in the Field of Intensive Ultrashort Pulses. Chem. Phys. 1990, 148, 33-45.

(20) Fainberg, B. D. Nonperturbative Analytic Approach to the Interaction of Intense Ultrashort Chirped Pulses with Molecules in Solution: Picture of "Moving” Potentials. J. Chem. Phys. 1998, 109, $4523-4532$.

(21) Fainberg, B. D.; Narbaev, B. Solvent-Controlled Theory Analysis of Chirped Pulse Excitation of Molecules in Solutions. J. Phys. Chem. B 2001, 105, 6085-6091.

(22) Fainberg, B. D.; Gorbunov, V. A. Coherent Population Transfer in Molecules Coupled with a Dissipative Environment by an Intense Ultrashort Chirped Pulse. J. Chem. Phys. 2002, 117, 7222-7232.

(23) Fainberg, B. D.; Gorbunov, V. A. Coherent Population Transfer in Molecules Coupled with a Dissipative Environment by Intense Ultrashort Chirped Pulse. II. A Simple Model. J. Chem. Phys. 2004, $121,8748-8754$.

(24) Gunaratne, T. C.; Zhu, X.; Lozovoy, V. V.; Dantus, M. Symmetry of Nonlinear Optical Response to Time Inversion of Shaped Femtosecond Pulses as a Clock of Ultrafast Dynamics. Chem. Phys. 2007, 338, 259-267.

(25) Torosov, B. T.; Vasilev, G. S.; Vitanov, N. V. Symmetries and Asymmetries in Coherent Atomic Excitation by Chirped Laser Pulses. Opt. Commun. 2010, 283, 1338-1345.

(26) Prokhorenko, V. I.; Nagy, A. M.; Waschuk, S. A.; Brown, L. S.; Birge, R. R.; Miller, R. J. D. Coherent Control of Retinal Isomerization in Bacteriorhodopsin. Science 2006, 313, 1257-1261.

(27) Prokhorenko, V. I.; Nagy, A. M.; Brown, L. S.; Miller, R. J. D. On the Mechanism of Weak-Field Coherent Control of Retinal Isomerization in Bacteriorhodopsin. Chem. Phys. 2007, 341, 296-309. (28) van der Walle, P.; Milder, M. T. W.; Kuipers, L.; Herek, J. L. Quantum Control Experiment Reveals Solvation-Induced Decoherence. Proc. Natl. Acad. Sci. U.S.A. 2009, 106, 7714-7717.

(29) Wand, A.; Kallush, S.; Shoshanim, O.; Bismuth, O.; Kosloff, R.; Ruhman, S. Chirp Effects on Impulsive Vibrational Spectroscopy: A Multimode Perspective. Phys. Chem. Chem. Phys. 2010, 12, 21492163.

(30) Katz, G.; Ratner, M. A.; Kosloff, R. Control by Decoherence: Weak Field Control of an Excited State Objective. New J. Phys. 2010, 12, 015003(1)-015003(13).

(31) Carson, E. A.; Diffey, W. M.; Shelly, K. R.; Lampa-Pastirk, S.; Dillman, K. L.; Schleicher, J. M.; Beck, W. F. Dynamic-Absorption Spectral Contours: Vibrational Phase-Dependent Resolution of LowFrequency Coherent Wave-Packet Motion of IR144 on the GroundState and Excited-State $\pi \rightarrow \pi^{*}$ Surfaces. J. Phys. Chem. A 2004, 108, 1489-1500. 
(32) Yu, A. C.; Tolbert, C. A.; Farrow, D. A.; Jonas, D. M. Solvatochromism and Solvation Dynamics of Structurally Related Cyanine Dyes. J. Phys. Chem. A 2002, 106, 9407-9419.

(33) Park, S.; Park, J. S.; Joo, T. Solvation Dynamics by Coherence Period Resolved Transient Grating. J. Phys. Chem. A 2011, 115, 39733979.

(34) Konar, A.; Shah, J. D.; Lozovoy, V. V.; Dantus, M. Optical Response of Fluorescent Molecules Studied by Synthetic Femtosecond Laser Pulses. J. Phys. Chem. Lett. 2012, 3, 1329-1335.

(35) Pastirk, I.; Resan, B.; Fry, A.; MacKay, J.; Dantus, M. No Loss Spectral Phase Correction and Arbitrary Phase Shaping of Regeneratively Amplified Femtosecond Pulses Using MIIPS. Opt. Express 2006, 14, 9537-9543.

(36) Coello, Y.; Lozovoy, V. V.; Gunaratne, T. C.; Xu, B. W.; Borukhovich, I.; Tseng, C. H.; Weinacht, T.; Dantus, M. Interference without an Interferometer: A Different Approach to Measuring, Compressing, and Shaping Ultrashort Laser Pulses. J. Opt. Soc. Am. B: Opt. Phys. 2008, 25, A140-A150. 